\title{
NONSTANDARD ANALYSIS AND AN APPLICATION TO THE SYMMETRIC GROUP ON NATURAL NUMBERS
}

\author{
MAURO DI NASSO AND YI ZHANG
}

\begin{abstract}
An introduction of nonstandard analysis in purely algebraic terms is presented. As an application, we give a nonstandard proof of a characterization theorem for compact subsets of $\operatorname{Sym}(\mathbb{N})$.
\end{abstract}

\section{INTRODUCTION}

Since their first introduction in the late 50's by A. Robinson, the methods of nonstandard analysis have been successfully applied to such diverse fields of mathematics as stochastic analysis, partial differential equations, Banach spaces, general topology, measure theory, mathematical finance, hydromechanics, etc. See [1] for an excellent and up-to-date collection of surveys. Probably the main point of the nonstandard approach to mathematics is the fact that it allows informal ideas and intuitions that are not directly tractable in a "standard" context, to correspond to actual mathematical objects. The infinitesimal numbers in real analysis are but a first basic example of this phenomenon.

The first section of this paper is a report of a seminar given by the first author at the Instanbul Bilg1 University, in April 2000. It consists of an elementary introduction to nonstandard analysis by purely algebraic means. The second section contains an application of nonstandard methods to the symmetric group on $\mathbb{N}$. Precisely, we give a characterization of the compact subsets of $\operatorname{Sym}(\mathbb{N})$, the space of permutations on $\mathbb{N}$ equipped with its natural topology, i.e. the pointwise convergence topology (see e.g. [4]). As a corollary, we get a proof that all compact subsets of $\operatorname{Sym}(\mathbb{N})$ have empty interior.

\section{NONSTANDARD ANALYSIS}

The most popular presentation of nonstandard analysis is the so-called superstructure approach (see [5] $\$ 4.4$ for a rigorous treatment of the foundational aspects). It is formulated in the language of mathematical logic, using notions such as "bounded quantifier formula" and "elementary embedding". In the sequel, we shall show that an alternative presentation of the ultrapower construction can be given in purely algebraic terms. As a consequence, we shall obtain the full strength of the superstructure approach by only using the basics of algebra.

We shall assume some familiarity with the notions of ring, maximal ideal, ring homomorphism, ordered field. An organic introduction to nonstandard methods is outside the scope of this paper, thus several intermediate results are stated without proofs or left as exercises that the reader is encouraged to work out in full detail (if

2000 Mathematics Subject Classification. 26E35; 20B30; 16S60; 03C20.

Work partially supported by Grant n. 40734 of Academy of Finland. 
needed, help can be found by consulting $[2,3])$. Excellent references for the various aspects of nonstandard analysis are the text books [9], [8], [7].

Let us denote by $\mathcal{F}(\mathbb{N}, \mathbb{R})$ the ring of real sequences $f: \mathbb{N} \rightarrow \mathbb{R}$ where operations are defined pointwise, i.e. $f+g$ is the sequence $h$ where $h(n)=f(n)+g(n)$ for all $n$, and similarly for the product $f \cdot g$. On $\mathcal{F}(\mathbb{N}, \mathbb{R})$ we consider the pointwise partial order $f \leq g \Leftrightarrow f(n) \leq g(n)$ for all $n$.

Definition 1.1. An ordered field ${ }^{*} \mathbb{R}$ is a hyperreal field if there is a ring homomorphism $\varphi$ from $\mathcal{F}(\mathbb{N}, \mathbb{R})$ onto $* \mathbb{R}$ :

$$
\varphi: \mathcal{F}(\mathbb{N}, \mathbb{R}) \rightarrow^{*} \mathbb{R}
$$

In particular, $\varphi$ is order-preserving. For simplicity, we identify each real number $r$ with $\varphi\left(c_{r}\right)$, the image of the corresponding constant sequence, so that $\mathbb{R} \subseteq{ }^{*} \mathbb{R}$. To avoid the trivial case, we also assume $\mathbb{R} \neq * \mathbb{R}$.

The existence problem is trivially solved by noticing that $* \mathbb{R}$ is a set of hyperreals if and only if it is isomorphic to a quotient of $\mathcal{F}$ modulo a maximal ideal $\mathcal{I}$. Now let the homomorphism $\varphi: \mathcal{F} \rightarrow^{*} \mathbb{R}$ be given.

Definition 1.2. A subset $X \subseteq \mathbb{N}$ is qualified if $X=\{n \mid f(n)=0\}$ for some real sequence $f$ with $\varphi(f)=0$.

We say that two sequences $f, g$ are equal almost everywhere, and write $f \sim g$, if they agree on a qualified set.

Similarly, we write $g \triangleleft f$ to mean that $g$ belongs to $f$ almost everywhere, i.e. if $\{n \mid f(n) \in g(n)\}$ is qualified.

By using the properties of ring homomorphism and the properties of field, the following can be proved.

\section{Proposition 1.3.}

(1) If $f(n) \neq g(n)$ for all $n$, then $\varphi(f) \neq \varphi(g)$.

(2) The family of qualified sets is closed under finite intersections.

(3) $\sim$ is an equivalence relation on the collection of all sequences.

(4) A set is qualified if and only if its complement is not.

(5) A superset of a qualified set is qualified.

(6) For all $f, g \in \mathcal{F}, f \sim g$ if and only if $\varphi(f)=\varphi(g)$.

(7) No finite set is qualified.

By using the language of set theory, items 2, 4, 5 and 7 above say that the family of qualified sets is a nonprincipal ultrafilter over $\mathbb{N}$.

We now want to extend the map $\varphi$ to other sequences of mathematical objects. To this end, in the following we shall concentrate on the mathematical universes grounded on $\mathbb{R}$ and $* \mathbb{R}$, respectively. They consist of $\mathbb{R}$ (of $* \mathbb{R}$, resp.), and of all its subsets, subsets of subsets, and so on. As usual in the literature, for sake of simplicity we assume that the hyperreal numbers are atoms, i.e. primitive entities that are not sets.

Definition 1.4. The standard universe is the superstructure over $\mathbb{R}$, i.e. the union $V(\mathbb{R})=\bigcup\left\{V_{n}(\mathbb{R}) \mid n \in \mathbb{N}\right\}$, where

$$
V_{0}(\mathbb{R})=\mathbb{R} ; \quad V_{n+1}(\mathbb{R})=V_{n}(\mathbb{R}) \cup\left\{X \mid X \subseteq V_{n}(\mathbb{R})\right\}
$$


The nonstandard universe is the superstructure over ${ }^{*} \mathbb{R}$.

We remark that virtually all objects of "standard" mathematics are (or can be coded as) elements of $V(\mathbb{R})$. For instance, in set theory an ordered pair $\langle a, b\rangle$ is usually identified with the Kuratowski pair $\{\{a\},\{a, b\}\}$. Thus a complex number, coded as an ordered pair of reals, is an element of $V_{2}(\mathbb{R})$; a real function $f: \mathbb{R} \rightarrow \mathbb{R}$, that can be identified with its graph $\{\langle x, y\rangle: y=f(x)\}$, is in $V_{3}(\mathbb{R})$; a space of real functions $\mathcal{S}$ is in $V_{4}(\mathbb{R})$; a topology on $\mathcal{S}$ is in $V_{6}(\mathbb{R})$, because it is a set of subsets of $\mathcal{S}$; etc. Similarly, all objects of "nonstandard" mathematics live in the nonstandard universe $V\left({ }^{*} \mathbb{R}\right)$. For every set $B$, denote by

$$
\mathcal{F}(\mathbb{N}, B) / \sim=\{[f] \mid f: \mathbb{N} \rightarrow B\} \text { where }[f]=\{g: \mathbb{N} \rightarrow B \mid f \sim g\}
$$

the quotient that consists of all $\sim$-equivalence classes of $B$-valued sequences. For each $n$ let $\mathcal{F}_{n}=\mathcal{F}\left(\mathbb{N}, V_{n}(\mathbb{R})\right) / \sim$.

Exercise 1.5. Prove that if $f \in \mathcal{F}_{n+1}$ and $g \triangleleft f$, then $g \in \mathcal{F}_{n}$.

We are now ready to extend the homomorphism $\varphi: \mathcal{F}(\mathbb{N}, \mathbb{R}) \rightarrow{ }^{*} \mathbb{R}$ to a mapping $\Psi: \bigcup_{n \in \mathbb{N}} \mathcal{F}_{n} \rightarrow V\left({ }^{*} \mathbb{R}\right)$.

Definition 1.6. By induction, let

- $\Psi([f])=\varphi(f)$ if $[f] \in \mathcal{F}_{0}$;

- $\Psi([f])=\{\Psi([g]) \mid g \triangleleft f\}$ if $[f] \in \mathcal{F}_{n+1}$.

By the previous exercise, the definition is well-posed. Clearly, $\Psi$ satisfies the equivalence $f \triangleleft g \Leftrightarrow \Psi([f]) \in \Psi([g])$.

Definition 1.7. The nonstandard embedding $*: V(\mathbb{R}) \rightarrow V\left({ }^{*} \mathbb{R}\right)$ is the mapping where ${ }^{*} B=\Psi\left(\left[c_{B}\right]\right)$ is the value under $\Psi$ of the $\sim$-equivalence class of the sequence constantly equal to $B .^{1}$

An object $B \in V(\mathbb{R})$ is called standard and ${ }^{*} B$ is called its nonstandard extension.

The nonstandard embedding preserves all "elementary" operations of sets, except the powerset operation $\wp(A)=\{a \mid a \subseteq A\}$.

Theorem 1.8 (Fundamental Theorem).

For all standard objects $B, C$ :

(1) ${ }^{*}(B \cup C)={ }^{*} B \cup{ }^{*} C$;

(2) ${ }^{*}(B \cap C)={ }^{*} B \cap{ }^{*} C$;

(3) ${ }^{*}(B \backslash C)={ }^{*} B \backslash{ }^{*} C$;

(4) $*\{B, C\}=\left\{{ }^{*} B,{ }^{*} C\right\}$;

(5) $*(B \times C)={ }^{*} B \times{ }^{*} C$;

(6) ${ }^{*}(\bigcup B)=\bigcup^{*} B$, i.e.

* $\{x \mid x \in y$ for some $y \in B\}=\left\{\xi \mid \xi \in \zeta\right.$ for some $\left.\zeta \in{ }^{*} B\right\}$;

(7) $*\{\langle x, x\rangle \mid x \in B\}=\left\{\langle\xi, \xi\rangle \mid \xi \in{ }^{*} B\right\}$;

$(8) *\{\langle x, y\rangle \mid x \in y \in B\}=\left\{\langle\xi, \zeta\rangle \mid \xi \in \zeta \in{ }^{*} B\right\}$;

\footnotetext{
${ }^{1}$ In other words, ${ }^{*} r=r$ if $r \in \mathbb{R}$ and ${ }^{*} B=\{\Psi([f]):[f] \in \mathcal{F}(\mathbb{N}, B) / \sim\}$ otherwise.
} 
(9) ${ }^{*}($ dom $B)=$ dom $^{*} B$, i.e.

${ }^{*}\{x \mid\langle x, y\rangle \in B$ for some $y\}=\left\{\xi \mid\langle\xi, \zeta\rangle \in{ }^{*} B\right.$ for some $\left.\zeta\right\} ;$

$(10) *(\operatorname{ran} B)=\operatorname{ran}^{*} B$, i.e.

${ }^{*}\{x \mid\langle x, y\rangle \in B$ for some $y\}=\left\{\xi \mid\langle\xi, \zeta\rangle \in{ }^{*} B\right.$ for some $\left.\zeta\right\} ;$

$(11) *\{\langle x, y\rangle \mid\langle y, x\rangle \in B\}=\left\{\langle\xi, \zeta\rangle \mid\langle\zeta, \xi\rangle \in{ }^{*} B\right\}$;

$(12) *\{\langle x, y, z\rangle \mid\langle x, z, y\rangle \in B\}=\left\{\langle\xi, \zeta, \sigma\rangle \mid\langle\xi, \sigma, \zeta\rangle \in{ }^{*} B\right\}$.

Clearly, $B$ and $C$ are assumed to be non-atoms, with the exception of item 4 .

Proof. (Hint.) First, prove the similar properties for the mapping $\Psi$. That is, if $h(n)=f(n) \cup g(n)$ almost everywhere, then $\Psi(h)=\Psi(f) \cup \Psi(g)$; etc.

Virtually every mathematical proposition can be expressed in terms of the twelve set-operations itemized above, called Gödel operations. Two basic examples are given in the following exercise.

Exercise 1.9. Prove that:

(1) If $f: A \rightarrow B$ is a function, then ${ }^{*} f:{ }^{*} A \rightarrow{ }^{*} B$ is a function with ${ }^{*} f\left({ }^{*} a\right)={ }^{*}[f(a)]$ for all $a \in A$. The similar property holds for functions of several variables. [Hint: Recall that $f$ is identified with its graph $\{\langle x, y\rangle \in A \times B \mid y=f(x)\}$.

$(2) \leq$ is a partial (or linear) ordering of the set $A$ if and only if its nonstandard extension $*(\leq)$ is a partial (or linear, resp.) ordering of ${ }^{*} A$. [Hint: Recall that $\leq$ is identified with the set of ordered pairs $\{\langle x, y\rangle \in A \times A \mid x \leq y\}$.]

Definition 1.10. The elements of $* \mathbb{N}$ are called hypernatural numbers and the elements of $* \mathbb{R}$ are called hyperreal numbers.

For sake of simplicity, when confusion is unlikely, some "asterisks" will be omitted. For instance, we shall use the same symbols + and $\cdot$ to denote the field operations on $\mathbb{R}$ and their nonstandard extensions on ${ }^{*} \mathbb{R}$. Similarly for the ordering $\leq$

Exercise 1.11. Prove that:

(1) $\langle\mathbb{N} ; \leq\rangle$ is a proper initial segment of $\left\langle{ }^{*} \mathbb{N} ; \leq\right\rangle$, i.e. if $x \in{ }^{*} \mathbb{N}$ and $x<y \in \mathbb{N}$, then also $x \in \mathbb{N}$.

(2) The set of unbounded or infinite hypernaturals $\mathbb{N}_{\infty}={ }^{*} \mathbb{N} \backslash \mathbb{N}$ is nonempty.

(3) $\langle * \mathbb{R} ;+; \cdot ; \leq\rangle$ is a real-closed field.

The infinitesimal numbers are those elements $\xi \in{ }^{*} \mathbb{R}$ such that $-r<\xi<r$ for all positive reals $r \in \mathbb{R}$ (e.g. the reciprocals $1 / \nu$ of infinite hypernaturals $\nu \in \mathbb{N}_{\infty}$.)

In practice, it is not convenient to apply the Fundamental Theorem 1.8 directly. In fact, it can be shown that any "elementary" property is true about standard objects $A_{1}, \ldots, A_{n}$ if and only if it is true about their nonstandard extensions ${ }^{*} A_{1}, \ldots,{ }^{*} A_{n}$. This fact, known in the literature as Liebniz principle, is the crucial tool of nonstandard analysis.

Theorem 1.12 (Liebniz principle).

An "elementary" mathematical statement $S\left(A_{1}, \ldots, A_{n}\right)$ is true if and only if the statement $S\left({ }^{*} A_{1}, \ldots,{ }^{*} A_{n}\right)$ is true. 
We remark that the Liebniz principle can be given a rigorous formulation by using the formalism of mathematical logic. ${ }^{2}$ In order to avoid a direct use of logic, here we presented only an informal version of it, grounded on the intuitive notion of "elementary" mathematical statement.

In some sense, we shall use Liebniz principle in the same way as Church's thesis is used in recursion theory. Whenever a "constructive" procedure to compute a function is indicated, by Church's thesis it can be directly assumed that such a function is recursive. In practice, there is no need to show explicitly how the function is obtained from the primitive recursive functions; it is enough to know that this is in principle possible.

Similarly, suppose we are considering some "elementary" property. Then we can directly apply the Liebniz principle and conclude that the given statement holds about some given standard objects if and only if it holds about the corresponding nonstandard extensions. Although this equivalence could be directly proved by repeatedly applying the preservation properties of the Fundamental Theorem 1.8, we do not need to do it in practice. ${ }^{3}$

Since we want to keep the notion of "elementary" statement at an informal level, it is crucial here to give some precise indications on the use of quantifiers. In fact - roughly speaking - a property is elementary if the scope of the existential and universal quantifiers is "bounded" by some set. A quantifier is bounded when it occurs in the form "for every $x \in X$ " or "there exists $y \in Y$ ", for some specified set $X$ or $Y$.

Let us try to clarify this crucial notion of bounded quantifier with an example. The minimum principle is usually expressed in the form: "Every (non-empty) finite set of natural numbers has a greatest element". This formulation is not "elementary" because the universal quantifier is not explicitly "bounded" by any set. However, an elementary re-formulation is easily found: "Every (non-empty) element of $\operatorname{Fin}(\mathbb{N})$ has a greatest element", with $\operatorname{Fin}(\mathbb{N})$ the collection of all finite subsets of $\mathbb{N}$. Now we can correctly apply the Leibniz principle and get: "Every element of ${ }^{*} \operatorname{Fin}(\mathbb{N})$ has a greatest element". The crucial point here is that $\operatorname{Fin}\left({ }^{*} \mathbb{N}\right)$ and ${ }^{*} \operatorname{Fin}(\mathbb{N})$ are different (the latter also contains some infinite subset of $* \mathbb{N}$ !). What the Liebniz principle actually proves is that the elements of $\operatorname{Fin}(\mathbb{N})$ - namely the hyperfinite subsets of *N - satisfy the same "elementary" properties as the finite subsets of $\mathbb{N}$.

We stress that in practice, restricting to "elementary" properties is not a limitation. In fact, virtually all mathematical statements can be equivalently re-phrased in "elementary" terms. For instance, let us consider the completeness property of the real numbers: "Every nonempty bounded subset of $\mathbb{R}$ has a least upper bound and a greatest lower bound". In order to make this property "elementary" we simply have to "bound" the existential quantifier and re-formulate: "Every non-empty bounded element of $\wp(\mathbb{R})$ has a l.u.b. and a g.l.b.". By the Leibniz principle, we get: "Every non-empty bounded element of *ø(R) has a l.u.b. and a g.l.b.".

\footnotetext{
${ }^{2}$ Namely, the Liebniz principle states a transfer property of the nonstandard embedding $*$ for bounded quantifier formulas in the first-order language of set theory. See [5] §4.4.

${ }^{3} \mathrm{~A}$ proof of the equivalence between the fundamental theorem and the Liebniz principle can be found in [3].
} 
We remark that two mathematical structures that satisfying the same "elementary" properties need not be isomorphic. This is in fact the very essence of nonstandard analysis. For instance, the real numbers and the hyperreal numbers are clearly not isomorphic, because e.g. $\mathbb{R}$ is complete but ${ }^{*} \mathbb{R}$ is not. ${ }^{4}$ However, $\mathbb{R}$ and ${ }^{*} \mathbb{R}$ are elementarily equivalent, i.e. they cannot be distinguished by any "elementary" property, by the Leibniz principle.

Definition 1.13. An object $x$ is internal if $x \in A^{*}$ for some $A$. An object of the nonstandard universe that is not internal is external.

Proposition 1.14 (Internal Definition Principle).

If $S\left(x, x_{1}, \ldots, x_{n}\right)$ is an "elementary" property and $B, B_{1}, \ldots, B_{n}$ are internal, then also the set $\left\{x \in B \mid S\left(x, B_{1}, \ldots, B_{n}\right)\right\}$ is internal.

Exercise 1.15. Prove that:

(1) Any nonstandard extension * $A$ is an internal set.

(2) If $A$ is internal and $a \in A$, then $a$ is internal.

(3) For every standard set $A,{ }^{*} \wp(A)=\left\{a \subseteq{ }^{*} A \mid a\right.$ is internal $\}$.

(4) If $\mathcal{F}(A, B)$ is the set of all functions from $A$ to $B$, then ${ }^{*} \mathcal{F}(A, B)=\{f$ : ${ }^{*} A \rightarrow{ }^{*} B \mid f$ is internal $\}$.

(5) The family of internal sets is closed under finite subsets, finite ordered tuples, finite Cartesian products, finite unions, finite intersections, and under complements.

The notion of internal set helps to correctly apply the Leibniz principle. For instance, by the previous exercise, the statement: "Every nonempty bounded subset of $\mathbb{N}$ has a greatest element" can be transferred to: "Every nonempty internal bounded subset of *N has a greatest element". As a consequence, the subset $\mathbb{N}_{\infty} \subset$ $* \mathbb{N}$ of infinite hypernatural numbers is external, because it is without greatest element. Then, also its complement $\mathbb{N}={ }^{*} \mathbb{N} \backslash \mathbb{N}_{\infty}$, the collection of standard hypernaturals, is external. Similarly, a statement of the form: "Every function $f: A \rightarrow B \ldots$.. transfers to: "Every internal function $f:{ }^{*} A \rightarrow{ }^{*} B \ldots$...

To summarize, we can say - roughly speaking - that when talking about subsets or about functions, then the property is transferred only to the corresponding internal subsets or functions, respectively.

We warn the reader that getting familiar with the distinction between internal and external objects is probably the hardest step in the learning process of nonstandard methods.

Another crucial tool of nonstandard analysis is the saturation principle. Recall that a (nonempty) family $\mathcal{B}$ of sets has the finite intersection property (FIP) if for finitely many $B_{1}, \ldots, B_{n} \in \mathcal{B}$, the intersection $B_{1} \cap \ldots \cap B_{n} \neq \emptyset$ is nonempty.

Theorem 1.16 (Saturation principle).

Suppose $\mathcal{B} \subseteq V_{n}\left({ }^{*} \mathbb{R}\right)$ is a countable family of internal sets with the FIP. Then $\bigcap \mathcal{B} \neq \emptyset$.

\footnotetext{
${ }^{4}$ For instance, the set of infinitesimals is bounded without least upper bound.
} 
Proof. Let $\mathcal{B}=\left\{b_{i} \mid i \in \mathbb{N}\right\}$. For each $i$, pick $f_{i} \in \mathcal{F}_{n}$ with $b_{i}=\Psi\left(\left[f_{i}\right]\right)$; and for every $k$, pick $x_{k} \in \bigcap_{i=0}^{k} b_{i}$. Without loss of generality we can assume that $x_{k}=\Psi\left(\left[g_{k}\right]\right)$ where $g_{k}(n) \in f_{i}(n)$ for all $n$ and for all $i=0, \ldots, k$.

Define $\vartheta(k)=g_{k}(k)$. Clearly $\vartheta \in \mathcal{F}_{n}$. For every $i, \vartheta \triangleleft f_{i}$ because $\{k: \vartheta(k) \in$ $\left.f_{i}(k)\right\} \supseteq\{k: k \geq i\}$ is cofinite, hence qualified. We conclude that $\Psi([\vartheta]) \in \cap \mathcal{B}$.

\section{Exercise 1.17.}

(1) No (infinite) countable set is internal.

(2) No (infinite) countable subset of $* \mathbb{N}$ is unbounded.

(3) ${ }^{*} A=\left\{{ }^{*} a \mid a \in A\right\}$ if and only if $A$ is finite. In particular, if $A$ is a finite set of atoms, then ${ }^{*} A=A$.

(4) For every infinite set $A$, there are external subsets $X \subset{ }^{*} A$, hence ${ }^{*} \wp(X) \neq$ $\wp\left({ }^{*} X\right)$.

We conclude this section with a comment about the uniqueness problem. We already pointed out that there are as many hyperreal fields $* \mathbb{R}$ as maximal ideals of $\mathcal{F}(\mathbb{N}, \mathbb{R})$. Thus, in principle, there is plenty of hyperreal fields. Now, a consequence of saturation is that $\left\langle{ }^{*} \mathbb{R} ; \leq\right\rangle$ is an $\eta_{1}$-set. Recall that a linearly ordered set $\langle L ; \leq\rangle$ is an $\eta_{\alpha}$-set if:

- no subset of size $<\aleph_{\alpha}$ is unbounded (above or below) in $L$;

- for every $A, B \subset L$ of size $<\aleph_{\alpha}$ with $A<B$ (i.e. $a<b$ for all $a \in A$ and $b \in B$ ) there exists $x$ with $A<x<B$.

A classic result by E. Erdös, L. Gillman and M. Henriksen [6] states that any two real-closed fields that are $\eta_{\alpha}$-sets of size $\aleph_{\alpha}$ are isomorphic. Hence:

Theorem 1.18. Assume the continuum hypothesis. Then all sets of hyperreal numbers are isomorphic as ordered fields. ${ }^{5}$

\section{A characterization of COMPaCt Sets in $\operatorname{Sym}(\mathbb{N})$}

Let us briefly recall the basic nonstandard characterizations in topology. Let $X$ be a topological space. For sake of simplicity we identify each element $x \in X$ with its nonstandard extension ${ }^{*} x \in{ }^{*} X$, so that $X \subseteq{ }^{*} X$.

Definition 2.1. The monad of an element $x \in X$ is the intersection

$$
\mu(x)=\bigcap\left\{{ }^{*} I \mid I \text { is a neighborhood of } x\right\}
$$

We write $\xi \approx x$ to mean $\xi \in \mu(x)$. We can think of $\mu(x)$ as the collection of those elements of * $X$ that are "infinitely close" to $x$. For instance, if $X=\mathbb{R}$ then $\mu(0)$ is the set of infinitesimal numbers of * $\mathbb{R}$.

In the following we shall assume that $X$ has a countable base of neighborhoods, so that each monad is non-empty by saturation. We remark that the same nonstandard treatment is also possible in the general case of uncountable bases. ${ }^{6}$

\footnotetext{
${ }^{5}$ This fact was first pointed out by W.A.J. Luxemburg in his lecture notes on nonstandard analysis that circulated since the early sixties.

${ }^{6}$ Precisely, suppose the topology has a base of (uncountable) cardinality $\kappa$. Then it can be proved that there exist nonstandard embeddings originated by suitable ring homomorphisms $\varphi$ : $\mathcal{F}(\kappa, \mathbb{R}) \rightarrow^{*} \mathbb{R}$ that satisfy the saturation property for families of size up to $\kappa$. Hence, monads are non-empty. See [3].
} 
Exercise 2.2. Prove that a topological space $X$ is Hausdorff if and only if $\mu(x) \cap$ $\mu(y)=\emptyset$ for all $x \neq y$.

The fundamental nonstandard tools in topology are given in the following

Proposition 2.3. Let $A$ be a subset of a topological space $X$. Then:

(1) $A$ is open if and only if $\xi \approx x \in A \Rightarrow \xi \in *^{*} A$;

(2) $A$ is closed if and only if $x \in X$ and $x \approx \xi \in \in^{*} A \Rightarrow x \in A$;

(3) $A$ is compact if and only if $\xi \in{ }^{*} A \Rightarrow \xi \approx x$ for some $x \in A$.

Definition 2.4. An element $\xi \in{ }^{*} X$ is called near-standard if $\xi \approx x$ for some $x \in X$.

Thus a closed subset $A$ is compact if and only if every $\xi \epsilon^{*} A$ is near-standard.

Exercise 2.5. Prove that $X \subset \mathbb{R}$ is compact if and only if $X$ is closed and bounded.

Now let $\mathcal{F}(\mathbb{N})$ be the space of all functions $f: \mathbb{N} \rightarrow \mathbb{N}$ with the pointwise convergence topology. Recall that, by definition, the family

$$
\{\Gamma(f, n) \mid n \in \mathbb{N}\} \text { where } \Gamma(f, n)=\{g \in \mathcal{F}(\mathbb{N}) \mid g(n)=f(n)\}
$$

is a pre-base of neighborhoods of $f$. Thus a set $A$ is open if and only if

$$
A \supseteq\left\{g \in \mathcal{F}(\mathbb{N}) \mid g\left(n_{i}\right)=f\left(n_{i}\right) \text { for } i=1, \ldots, k\right\}
$$

for some function $f$ and finitely many $n_{1}, \ldots, n_{k} \in \mathbb{N}$. Clearly $\mathcal{F}(\mathbb{N})$ is Hausdorff. Now consider its nonstandard extension

$$
{ }^{*} \mathcal{F}(\mathbb{N})=\left\{\sigma:{ }^{*} \mathbb{N} \rightarrow{ }^{*} \mathbb{N} \mid \sigma \text { is internal }\right\}
$$

The following fact is proved in a straightforward manner.

Proposition 2.6. The monad of a function $f: \mathbb{N} \rightarrow \mathbb{N}$ is the set:

$$
\mu(f)=\left\{\sigma \in{ }^{*} \mathcal{F}(\mathbb{N}) \mid \sigma(n)=f(n) \text { for all } n \in \mathbb{N}\right\}
$$

In other words, the monad of $f$ consists of all internal functions $\sigma:{ }^{*} \mathbb{N} \rightarrow{ }^{*} \mathbb{N}$ extending $f$. Thus $\sigma \approx \tau$ if and only if $\sigma$ and $\tau$ agree on the standard natural numbers, i.e. when their restrictions $\sigma \uparrow \mathbb{N}=\tau \uparrow \mathbb{N}$ coincide. In particular, $\sigma$ is near-standard if and only if $\sigma(n) \in \mathbb{N}$ for each $n \in \mathbb{N}$.

Theorem 2.7. Let $K$ be a closed subset of $\mathcal{F}(\mathbb{N})$. Then $K$ is compact if and only if all sets $\Omega_{n}(K)=\{f(n) \mid f \in K\}$ are finite.

Proof. Since $K$ is closed, $K$ is compact if and only if every $\sigma \in{ }^{*} K$ is near-standard. By contradiction, assume that for some $n, \Omega_{n}(K)=\{f(n) \mid f \in K\}$ is infinite. Let $B_{k}=\{f \in K \mid f(n)>k\}$. The family $\mathcal{B}=\left\{B_{k} \mid k \in \mathbb{N}\right\}$ has the FIP, thus by saturation there is $\sigma \in \bigcap\left\{{ }^{*} B_{k} \mid k \in \mathbb{N}\right\}$. Clearly $\sigma(n) \notin \mathbb{N}$, hence $\sigma$ is not nearstandard.

Viceversa let $\sigma \in{ }^{*} K$. For each $n, \Omega_{n}(K)$ is finite, hence $\Omega_{n}(K)={ }^{*} \Omega_{n}(K)=$ $\left\{\tau(n) \mid \tau \in{ }^{*} K\right\} \subseteq \mathbb{N}$. In particular $\sigma(n) \in \mathbb{N}$. We conclude that $\sigma$ is near-standard. 
Corollary 2.8. Any compact subset in $\mathcal{F}(\mathbb{N})$ has empty interior. In particular, $\mathcal{F}(\mathbb{N})$ is not locally compact.

Proof. By contradiction, let $K$ be a compact set with

$$
K \supseteq\left\{f \in \mathcal{F}(\mathbb{N}) \mid f\left(n_{i}\right)=k_{i} \text { for } i=1, \ldots, s\right\}
$$

for some $n_{1}, \ldots, n_{s}, k_{1}, \ldots, k_{s} \in \mathbb{N}$. Fix $n \neq n_{i}$ for all $i$. For each $m$, pick $g_{m} \in \mathcal{F}(\mathbb{N})$ such that $g_{m}\left(n_{i}\right)=k_{i}$ for $i=1, \ldots, s$ and $g_{m}(n)=m$. Clearly $\left\{g_{m} \mid m \in \mathbb{N}\right\} \subseteq K$. Then $\Omega_{n}(K)=\mathbb{N}$ is infinite and $K$ is not compact.

Now let us consider $\operatorname{Sym}(\mathbb{N})$, the subspace of permutations of $\mathcal{F}(\mathbb{N})$ (a good reference on the symmetric group $\operatorname{Sym}(\mathbb{N})$ is [4]).

Proposition 2.9. $\operatorname{Sym}(\mathbb{N})$ is not closed.

Proof. Pick $\nu$ an unbounded hypernatural, and consider the internal permutation $\sigma:{ }^{*} \mathbb{N} \rightarrow{ }^{*} \mathbb{N}$ with $\sigma(i)=i+1$ for all $i<\nu ; \sigma(\nu)=0$ and $\sigma(i)=i$ for all $i>\nu$. Clearly $\sigma \approx f$ where $f(n)=n+1$ for all $n \in \mathbb{N}$, but $f \notin \operatorname{Sym}(\mathbb{N})$ is not a permutation.

Let us concentrate on $\operatorname{Sym}(\mathbb{N})$ as a topological space itself, with the topology inherited from $\mathcal{F}(\mathbb{N})$. Notice that $A$ is open in $\operatorname{Sym}(\mathbb{N})$ if and only if

$$
A \supseteq\left\{g \in \operatorname{Sym}(\mathbb{N}) \mid g\left(n_{i}\right)=f\left(n_{i}\right) \text { for } i=1, \ldots, k\right\}
$$

for some permutation $f$ and finitely many $n_{1}, \ldots, n_{k}$. Clearly, also $\operatorname{Sym}(\mathbb{N})$ is Hausdorff. An internal permutation $\sigma:{ }^{*} \mathbb{N} \rightarrow{ }^{*} \mathbb{N}$ is near-standard if and only if its restriction $\sigma \mid \mathbb{N}$ is a permutation on $\mathbb{N}$, i.e. if $\sigma(n) \in \mathbb{N}$ and $\sigma^{-1}(n) \in \mathbb{N}$ for all $n \in \mathbb{N}$. There is a characterization of the compact subsets of $\operatorname{Sym}(\mathbb{N})$ that is similar to the one proved for $\mathcal{F}(\mathbb{N})$.

Theorem 2.10. Let $K$ be a closed subset of $\operatorname{Sym}(\mathbb{N})$. Then $K$ is compact if and only if all sets $\Omega_{n}(K)=\{f(n) \mid f \in K\}$ and $\Lambda_{n}(K)=\left\{f^{-1}(n) \mid f \in K\right\}$ are finite.

Proof. If, by contradiction, $\Omega_{n}(K)$ is infinite for some $n$, then proceed as in the previous theorem. If there exists $n$ with $\Lambda_{n}(K)$ infinite, then by saturation there is $\sigma \in{ }^{*} K$ with $\sigma^{-1}(n)=\xi \notin \mathbb{N}$. But $\sigma:{ }^{*} \mathbb{N} \rightarrow{ }^{*} \mathbb{N}$ is a permutation, thus for all $m \in \mathbb{N}, m \neq \xi$ implies that $\sigma(m) \neq n$. In particular, the restriction $\sigma\lceil\mathbb{N}$ is not a permutation on $\mathbb{N}$ and $\sigma$ is not near-standard in $\operatorname{Sym}(\mathbb{N})$.

Vice versa, let the internal permutation $\sigma \in{ }^{*} K$ be given. Proceeding as in the previous theorem it is shown that $\sigma(n) \in \mathbb{N}$ and $\sigma^{-1}(n) \in \mathbb{N}$ for all $n \in \mathbb{N}$. Thus the restriction $\sigma \uparrow \mathbb{N}$ is a permutation on $\mathbb{N}$ and $\sigma$ is near-standard.

Corollary 2.11. Any compact subset in $\operatorname{Sym}(\mathbb{N})$ has empty interior. In particular, $\operatorname{Sym}(\mathbb{N})$ is not locally compact.

Proof. By contradiction, suppose $K$ is a compact set such that

$$
K \supseteq\left\{g \in \operatorname{Sym}(\mathbb{N}) \mid g\left(n_{i}\right)=f\left(n_{i}\right) \text { for } i=1, \ldots, k\right\}
$$

for some permutation $f$. Pick $n>\max \left\{n_{1}, \ldots, n_{k}, f\left(n_{1}\right), \ldots, f\left(n_{k}\right)\right\}$. For each $m$, let $g_{m}$ be a permutation on $\mathbb{N}$ with $g_{m}\left(n_{i}\right)=f\left(n_{i}\right)$ for $i=1, \ldots, k$ and $g_{m}(n)=$ $n+m$. Clearly each $g_{m} \in K$. Then $\Omega_{n}(K)$ is infinite, and $K$ is not compact. 
Acknowledgement. Mauro Di Nasso is grateful to the Bilg1 University for providing the friendly and stimulating environment where this joint work with Yi Zhang originated.

\section{REFERENCES}

[1] L.O. Arkeryd, N.J. Cutland and C.W. Henson, eds., Nonstandard Analysis; Theory and Applications, NATO ASI Series C, vol. 493, Kluwer Academic Publishers, 1997.

[2] V. Benci, An algebraic approach to nonstandard analysis, in Calculus of Variations and Partial Differential Equations (G. Buttazzo, A. Marino and M.K.V. Murthy, eds.), Springer (1999), 285-326.

[3] V. Benci and M. Di Nasso, A ring homomorphism is enough to get nonstandard analysis, submitted.

[4] P.J. Cameron, Oligomorphic Permutation Groups, Cambridge University Press, 1990.

[5] C.C. Chang and H.J. Keisler, Model Theory (3rd edition), North-Holland, 1990.

[6] P. Erdös, L. Gillman and M. Henriksen, An isomorphism theorem for real-closed fields, Annals of Mathematics 61 (1955), 542-554.

[7] R. Goldblatt, Lectures on the Hyperreals: an Introduction to Nonstandard Analysis, Springer, New York, 1998.

[8] A. Hurd and P.A. Loeb, An Introduction to Nonstandard Real Analysis, Academic Press, New York, 1985

[9] K. Stroyan and W.A.J. Luxemburg, Introduction to the Theory of Infinitesimals, Academic Press, New York, 1976.

Dipartimento di Matematica Applicata "U. Dini", Università di Pisa, Italy,

E-mail address: dinasso@dma.unipi.it

Department of Mathematics, University of Helsinki, Finland.

E-mail address: zhang@mat-92.pc.helsinki.fi 